\title{
Erratum
}

\section{A simple model for doublet bands in doubly odd nuclei}

N. Yoshinaga ${ }^{1, a}$ and K. Higashiyama $a^{2,3}$

1 Department of Physics, Saitama University, Saitama City 338-8570, Japan

2 Department of Physics, Chiba Institute of Technology, Narashino, Chiba 275-0023, Japan

3 Department of Physics, University of Tokyo, Hongo, Tokyo 113-0033, Japan

Original article: Eur. Phys. J. A 30, 343-346 (2006) DOI: 10.1140/epja/i2006-10141-6

Received: 28 February 2007

Published online: 29 March 2007 - C Società Italiana di Fisica / Springer-Verlag 2007

The paper contains the following errors:

1) Equation (9) on p. 344 should read

$$
T(M 1)=\sqrt{\frac{3}{4 \pi}}\left(g_{c} R+g_{\ell \nu} \ell_{\nu}+g_{s \nu} s_{\nu}+g_{\ell \pi} \ell_{\pi}+g_{s \pi} s_{\pi}\right) .
$$

2) Equation (14) on p. 345 should read

$$
g_{c}=\mu\left(2_{1}^{+}\right) / 2 .
$$

3) In the third line of the right column on p. 345 "negative" should read "positive".

All calculations of the paper have been done with the correct equations.

\footnotetext{
a e-mail: yoshinaga@phy.saitama-u.ac.jp
} 